\title{
Finite elements numerical solution to deep beams based on layerwise displacement field
}

\author{
M. M. S. Vilar $^{1} \cdot$ M. Sartorato ${ }^{1,2} \cdot$ H. B. Santana ${ }^{1} \cdot$ M. R. Leite ${ }^{1}$
}

Received: 12 December 2017 / Accepted: 30 August 2018/Published online: 11 September 2018

(C) The Brazilian Society of Mechanical Sciences and Engineering 2018

\begin{abstract}
This paper proposes a numerical solution to deep beams using the layerwise displacement theory. Most of the methods for performing structural analyses of deep beams have geometric and boundary conditions limitations, as well as modeling inconveniences. This paper provides a finite element solution for deep beams based on a layerwise displacement field considering the full stress/strain tensors. In this formulation, the cross section is discretized in a pre-defined number of independent virtual layers, with linear interpolation within the thickness direction. To validate the model developed, two numerical examples are analyzed. The first is a reinforced concrete deep beam with two supports, loaded over the top face, validated by finite element analysis based on solid-element ABAQUS ${ }^{\mathrm{TM}}$ software. Next, an isotropic deep beam with both ends cantilevered is analyzed and the outcome is compared to the literature. The results of both numerical examples are accurate and can estimate the complete state of stress over all domains of the element. Moreover, the layerwise formulation does not suffer from shear and membrane locking, and it may use fewer computational resources than equivalent 3D finite element analyses.
\end{abstract}

Keywords Layerwise $\cdot$ Deep beam · Numerical solution $\cdot$ Finite elements

\section{Introduction}

Deep beams are heavily loaded structural members that can be found in numerous structures, such as transfer girders in buildings, cap beams in bridge bents, pile caps in foundations, and other cumbersome elements. Due to their geometry, deep beams can carry very large shear forces when compared to slender regular beams. Therefore, the classical linear assumptions, such as the Bernoulli beams and Timoshenko, beam are no longer applicable, as they do not accurately account for out-of-plane shear distortions. The American code for concrete design ACI 318 [1]

Technical Editor: Paulo de Tarso Rocha de Mendonça.

M. M. S. Vilar

vilarmms@gmail.com

1 Group of Aeronautic Structure (GEA), Department of Aeronautical Engineering, School of Engineering of São Carlos, University of São Paulo, Av. João Dagnone, 1100 Jardim Santa Angelina, São Carlos, SP 13563-120, Brazil

2 São Paulo State University (UNESP), São João da Boa Vista Campus, Av. Profa. Isette Corrêa Fontão, 505 - Jardim das Flores, São João da Boa Vista, SP 13876-750, Brazil demands that the strain field of deep beams should be nonlinear, and it suggests the strut and tie method (STM) approach to analyze deep beams. Furthermore, several design codes, such as the European, Canadian and Brazilian codes, also permit deep beam analyses via the STM.

The STM reduces the complex case of a solid under flexure and shear to a simple truss model, in which the diagonal struts represent the compressive stress field and the ties represent the tensile stress on reinforcements [2]. There have been many improvements to the STM theory, such as the semi-empirical model proposed by Russo et al. [3], and the analytical two-parameter kinematic theory of Mihaylov et al. [4]. However, the STM theory still presents conservative results [5]. In addition, the STM disregards the complete tri-axial state of stress/strain on deep beams, and it can be difficult to design trusses in complex cases, such as cutouts and deep beams with unusual boundary conditions. For example, the STM approach is incapable of solving a cantilever deep beam problem.

Numerical models have been developed to perform structural analyses of deep beams. These methods are based on finite element procedures, the finite-difference method, and other numerical approximations. The 
numerical models are accurate but usually demand a high computational cost. The special feature of numerical methods is the flexibility to model more complex cases. For instance, Ahmed and Idris [6] proposed a finite-difference technique based on a displacement function to solve homogeneous deep beams with both ends fixed subjected to a constant load. Later, Ahmed et al. [7] extended the same approach to cantilever beams subjected to a distributed shear force at the free end. However, no attempt has been made to analyze cantilevered deep beams considering a tri-axial state of stress.

This paper proposes a finite element formulation with a layerwise displacement-based theory to perform structural analyses on deep beams. In this theory, the cross section is discretized in virtual layers, the elastic properties of which may be different to each other. Hence, this approach is well suited to heterogeneous materials, such as reinforced concrete, or to progressive damage analyses of any kind of beam. The layerwise theory was introduced to 3D general plate elements by Reddy [8] and it can be easily applied to deep beams. The main advantages of the layerwise theory are the fact that this formulation is able to predict the correct stress/strain field with the need for fewer computational resources across all components when compared to an equivalent 3D solid analysis. In addition, it can capture the discontinuity of stresses and strains at the interface of two different materials [9], which is not possible in the equivalent single layer (ESL) theories and shear deformation theory (SDT). Furthermore, the layerwise theory is free of shear and membrane locking [10] and one can insert more complex boundary conditions not modeled through the STM or ESL theories.

\section{Methodology}

A numerical finite element model based on the layerwise displacement theory is developed for deep beams. In this displacement theory, the element is discretized through the thickness in multiple virtual layers. Furthermore, two numerical models are presented in order to validate the formulation. The first is a reinforced deep beam simulated in ABAQUS ${ }^{\mathrm{TM}}$ software using a solid-element formulation with quadratic interpolation in all directions. In this solid model, two approaches have been considered for the simulation of the steel bars: modeling the bars as heterogynous phases and modeling the bars as homogenized layers with elastic properties that differ from pure concrete. The second numerical example is an isotropic deep beam with both ends cantilevered, solved through the $2 \mathrm{D}$ finite-difference technique [6].

\section{Layerwise theory review}

The SDT is commonly used to approximate the displacement field of structural analyses of plate and shell elements. In the SDT, the continuity of the distribution of the displacement components $\left(u_{1}, u_{2}, u_{3}\right)$ is assumed. Consequently, this assumption fails to accurately calculate the out-of-plane stresses on interfaces between two different materials, and it also fails to present the zigzag behavior of the displacement field throughout the element thickness of heterogeneous-layered materials [9]. Moreover, the stresses over the interface of two different materials should be continuous, which may not occur in the SDT [11]. In order to avoid the SDT's limitations it is necessary to discretize each material separately. In case of a laminated material, this discretization process can be done by discretizing the materials over the thickness as an assembly of individual layers. Figure 1 illustrates the difference between the layerwise theory and the ESL theories of the displacement field throughout the thickness of a composite material whose elastic properties differ in each layer.

In the layerwise theory developed by Reddy [8], the displacement field is a linear combination of two independent classes of function, one function one function of the thickness coordinate and the other in function of the plane, as demonstrated in Eq. (1)

$u_{i}(x, y, z)=u_{i}^{0}(x, y)+\sum_{j=1}^{n} u_{i}^{j}(x, y) \phi_{j}(z)$

where " $n$ " is the number of analysis layers discretized across the thickness, and $\phi_{j}$ are the function over thickness called the layerwise function. In order to guarantee the compatibility between layers, the layerwise function should be continuous in each layer and must converge at interfaces.

Layerwise theories require more computational effort than ESL theories, especially when the cross section is discretized using several layers. However, Reddy [11] stated that layerwise theories can demand fewer computational resources when compared to equivalent full 3D solid analyses. Hence, it can be more attractive to analyze deep beams using layerwise theory, rather than $3 \mathrm{D}$ solid formulations. Furthermore, layerwise theories can predict the correct out-of-plane stresses between two different materials, which may be interesting for modeling reinforced concrete deep beams, or homogeneous deep beams with stiffeners, or for making design decisions using damage analysis. 
Fig. 1 Schematics of different deformation theories: a linear ESL; b higher-order ESL; c linear layerwise; $\mathbf{d}$ higherorder layerwise

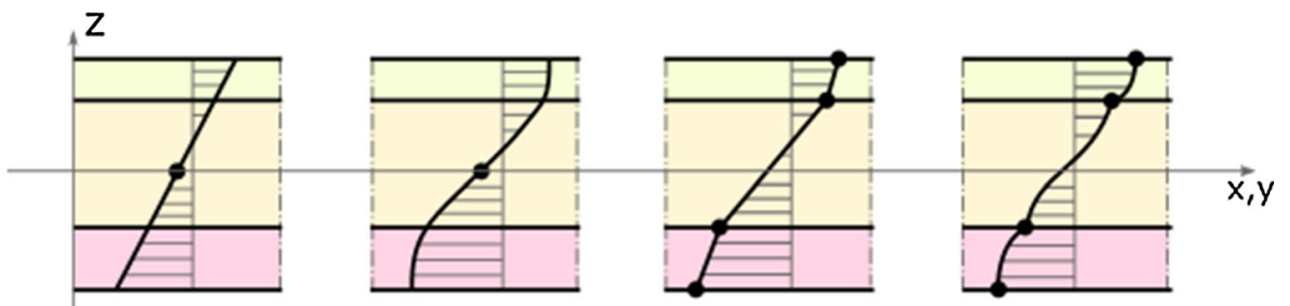

(a)

(b)

\section{Governing equation}

The governing equation of the mathematical problem is shown in Eq. (2) (sum in $k$ ),

$$
\begin{aligned}
& \int_{\Omega^{(k)}} \int_{Z_{\text {bot }}^{(k)}}^{Z_{\text {top }}^{(k)}} \delta \varepsilon^{(k)^{T}} \sigma^{(k)} \mathrm{d} z \mathrm{~d} \Omega^{(k)} \\
& =\left.\int_{\Omega^{(k)}} \delta u_{i}^{(k)} P_{\text {top } i}^{(k)}\right|_{Z=Z_{\text {top }}} \mathrm{d} \Omega^{(k)}+\left.\int_{\Omega^{(k)}} \delta u_{i}^{(k)} P_{\mathrm{bot} i}^{(k)}\right|_{Z=Z_{\text {bot }}} \mathrm{d} \Omega^{(k)} \\
& +\oint_{\Gamma_{t}^{(k)}} \int_{Z_{\text {bot }}^{(k)}}^{Z_{\text {top }}^{(k)}} \delta u_{i}^{(k)} t_{i}^{(k)} \mathrm{d} z \mathrm{~d} \Gamma_{t}^{(k)}+\left.\delta u_{i} F_{i}\right|_{\Gamma_{f}}
\end{aligned}
$$

where $k=1 \ldots n$ is the index for the discretized layers of the material and $i$ is the index for the coordinate system directions. $P_{i}^{k}$ are pressures that may be applied to the top or bottom of each layer, $t_{i}^{k}$ are the Cauchy stresses applied to the edges of the plate on each layer over a subdomain $\Gamma_{t}^{k} \in \Omega^{k}$ of the element in which the stresses are specified, and $F_{i}$ are concentrated forces applied to specific nodes in the $T_{f}$ set. It is assumed that $\Omega^{k}=\Omega$, i.e., the areas of all layers are considered to be the same before deformation. All domains and sub-domains are demonstrated in Fig. 2.

The relation between stresses and strains follows the classical form of Hooke's law. However, it is assumed that the virtual layers with only concrete material have an isotropic relation while the homogenized virtual layers (layers with steel bars) are transversely isotropic. Hence, the constitutive law selected is the transversely isotropic materials shown in Eqs. (3) and (4). Note that the constitutive relation shown is already in terms of the global reference plane. Demasi [12] demonstrates the procedure for changing from a general local system to the global reference. (c) (d)

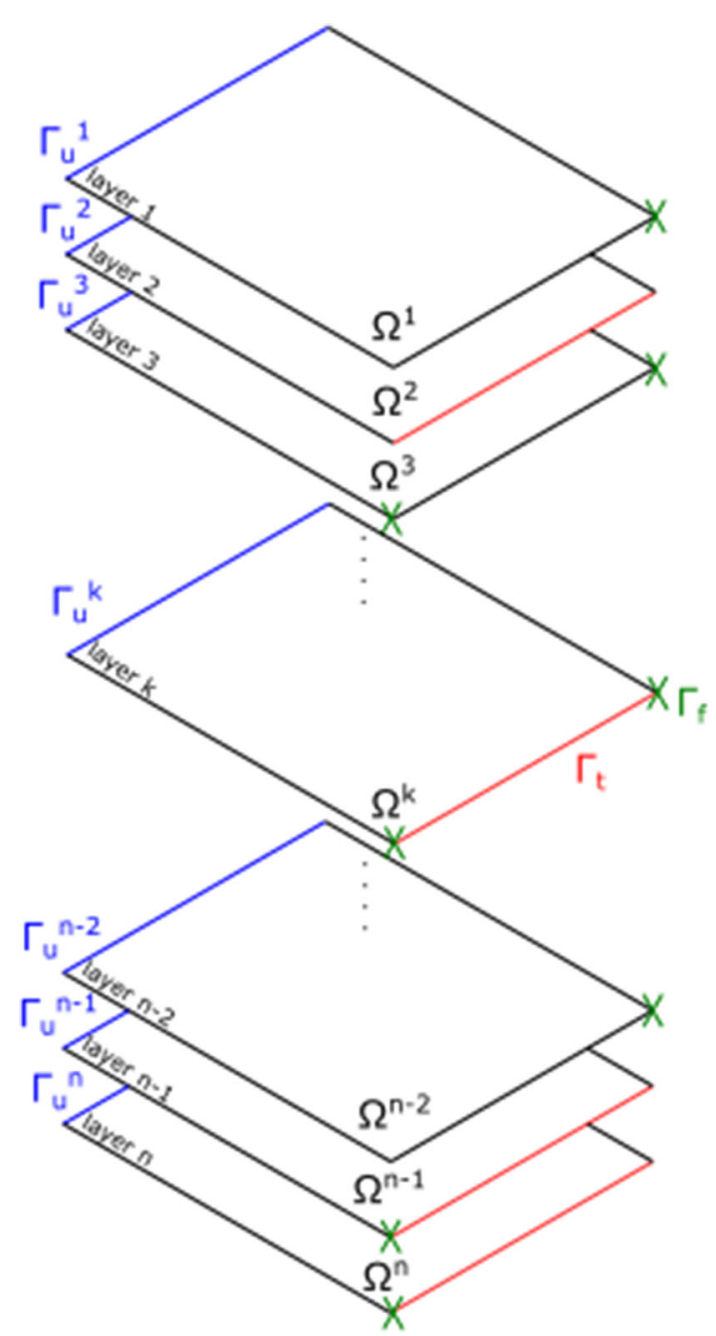

Fig. 2 Illustration of the domains and sub-domains of the governing equation

$$
\left\{\begin{array}{l}
\sigma_{11} \\
\sigma_{22} \\
\tau_{12} \\
\tau_{13} \\
\tau_{23} \\
\sigma_{33}
\end{array}\right\}=\left[\begin{array}{cccccc}
C_{11} & C_{12} & 0 & 0 & 0 & C_{12} \\
C_{12} & C_{22} & 0 & 0 & 0 & C_{23} \\
0 & 0 & C_{33} & 0 & 0 & 0 \\
0 & 0 & 0 & C_{33} & 0 & 0 \\
0 & 0 & 0 & 0 & C_{33} & 0 \\
C_{12} & C_{23} & 0 & 0 & 0 & C_{22}
\end{array}\right]\left\{\begin{array}{l}
\varepsilon_{11} \\
\varepsilon_{22} \\
\gamma_{12} \\
\gamma_{13} \\
\gamma_{23} \\
\varepsilon_{33}
\end{array}\right\}
$$

where 
$C_{11}=E_{11}\left(1-v_{23} v_{32}\right) / \Delta ; C_{12}=E_{11}\left(v_{21}-v_{23} v_{31}\right) / \Delta$

$C_{22}=E_{22}\left(1-v_{13} v_{31}\right) / \Delta ; C_{23}=E_{11}\left(v_{32}-v_{12} v_{31}\right) / \Delta ;$

$C_{33}=G_{12} ; \Delta=1-\left(v_{23} v_{32}+v_{12} v_{21}+v_{13} v_{31}+2 v_{21} v_{32} v_{13} ;\right.$

$v_{i j} E_{j j}=v_{j i} E_{i i} \quad i=1,2,3$

The specific internal energy term of the governing equation may be written as in Eq. (5):

$$
\begin{aligned}
\delta \varepsilon^{(k)^{T}} \sigma^{(k)}= & +\delta \varepsilon_{11}^{(k)} C_{11}^{(k)} \varepsilon_{11}^{(k)}+\delta \varepsilon_{11}^{(k)} C_{12}^{(k)} \varepsilon_{22}^{(k)}+\delta \varepsilon_{11}^{(k)} C_{12}^{(k)} \varepsilon_{33}^{(k)} \\
& +\delta \varepsilon_{22}^{(k)} C_{12}^{(k)} \varepsilon_{11}^{(k)}+\delta \varepsilon_{22}^{(k)} C_{22}^{(k)} \varepsilon_{22}^{(k)}+\delta \varepsilon_{22}^{(k)} C_{23}^{(k)} \varepsilon_{33}^{(k)} \\
& +\delta \gamma_{12}^{(k)} C_{33}^{(k)} \gamma_{12}^{(k)}+\delta \gamma \varepsilon_{13}^{(k)} C_{33}^{(k)} \gamma_{13}^{(k)}+\delta \gamma_{23}^{(k)} C_{33}^{(k)} \gamma_{23}^{(k)} \\
& +\delta \varepsilon_{33}^{(k)} C_{12}^{(k)} \varepsilon_{11}^{(k)}+\delta \varepsilon_{33}^{(k)} C_{23}^{(k)} \varepsilon_{22}^{(k)}+\delta \varepsilon_{33}^{(k)} C_{22}^{(k)} \varepsilon_{33}^{(k)}
\end{aligned}
$$

\section{Model discretization}

The element used in this model is the homogeneous 4-node rectangular model, with linear layerwise interpolation over the thickness. The displacement field of the continuum is shown in Eq. (6) and its approximation via finite element is shown in Eq. (7).

$u_{i}^{(k)}(x, y, z)=u_{i}^{(k)}(x, y) \phi_{1}(z)+u_{i}^{k+1}(x, y) \phi_{2}(z)$

$u_{i}^{(k)}(x, y, z)=\sum_{j=1}^{4} N_{j}(x, y)\left[u_{i}^{(k)}(x, y) \phi_{1}(z)+u_{i}^{(k+1)}(x, y) \phi_{2}(z)\right]$

where " $i$ " are the three directions of the Cartesian system; " $k$ " is the layer; " $\phi_{1}$ " and " $\phi_{2}$ " are the 2-node shape functions of the bottom and top of the layer, respectively,

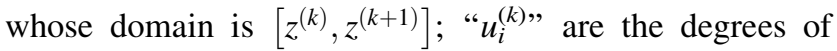
freedom of the layer; and " $N_{j}$ " are the shape functions of the standard 4-node element.

The total amount of degrees of freedom of a single node depends upon the number of virtual layers discretized. For $k$ layers, the vector of degrees of freedom of the $j$-th node is shown in Eq. (8):

$u_{j}=\left[u_{1}^{(1)} u_{2}^{(1)} u_{3}^{(1)} u_{1}^{(2)} u_{2}^{(2)} u_{3}^{(2)} \ldots u_{1}^{(k-1)} u_{2}^{(k-1)} u_{3}^{(k-1)} u_{1}^{(k)} u_{2}^{(k)} u_{3}^{(k)}\right]_{j}$

Considering a linear compatibility of the strain-displacement relation, the strain field of the $j$-th node is demonstrated in Eq. (7).

$$
\begin{aligned}
\varepsilon_{i i}^{(k)}= & \frac{\partial N_{j}}{\partial X_{i}}\left[u_{i}^{(k)(j)} \phi_{1}+u_{i}^{(k+1)(j)} \phi_{2}\right] ; \quad i=1,2 \\
\varepsilon_{33}^{(k)}= & N_{j}\left[u_{3}^{(k)(j)} \frac{\partial \phi_{1}}{\partial X_{3}}+u_{3}^{(k+1)(j)} \frac{\partial \phi_{2}}{\partial X_{3}}\right] \\
\gamma_{12}^{(k)}= & \frac{\partial N_{j}}{\partial X_{2}}\left[u_{1}^{(k)(j)} \phi_{1}+u_{1}^{(k+1)(j)} \phi_{2}\right]+\frac{\partial N_{j}}{\partial X_{1}}\left[u_{2}^{(k)(j)} \phi_{1}+u_{2}^{(k+1)(j)} \phi_{2}\right] \\
\gamma_{i 3}^{(k)}= & N_{j}\left[u_{3}^{(k)(j)} \frac{\partial \phi_{1}}{\partial X_{3}}+u_{3}^{(k+1)(j)} \frac{\partial \phi_{2}}{\partial X_{3}}\right]+\frac{\partial N_{j}}{\partial X_{i}}\left[u_{3}^{(k)(j)} \phi_{1}+u_{3}^{(k+1)(j)} \phi_{2}\right] ; \\
& i=1,2
\end{aligned}
$$

Finally, the strain-displacement relation can be derived by means of the matrix displayed in Eq. (10) (named here $B$ matrix). It is important to mention that the $B$ matrix relates to a specific virtual lamina of an element; consequently, it is necessary to assemble the $B$ matrices over the thickness to retrieve the stiffness matrix of an element. This process of assemble over thickness is discussed in more detail in [13] and is illustrated in relation to a twolayered simple bar element in Figs. 3 and 4.

$[B]_{j}^{k}=\left[\begin{array}{cccccc}\frac{\partial N_{j}}{\partial X_{1}} \phi_{1} & 0 & 0 & \frac{\partial N_{j}}{\partial X_{1}} \phi_{2} & 0 & 0 \\ 0 & \frac{\partial N_{j}}{\partial X_{2}} \phi_{1} & 0 & 0 & \frac{\partial N_{j}}{\partial X_{2}} \phi_{2} & 0 \\ \frac{\partial N_{j}}{\partial X_{2}} \phi_{1} & \frac{\partial N_{j}}{\partial X_{1}} \phi_{1} & 0 & \frac{\partial N_{j}}{\partial X_{2}} \phi_{2} & \frac{\partial N_{j}}{\partial X_{1}} \phi_{2} & 0 \\ N_{j} \frac{\partial \phi_{1}}{\partial X_{3}} & 0 & \frac{\partial N_{j}}{\partial X_{1}} \phi_{1} & N_{j} \frac{\partial \phi_{2}}{\partial X_{3}} & 0 & \frac{\partial N_{j}}{\partial X_{1}} \phi_{2} \\ 0 & N_{j} \frac{\partial \phi_{1}}{\partial X_{3}} & \frac{\partial N_{j}}{\partial X_{2}} \phi_{1} & 0 & N_{j} \frac{\partial \phi_{2}}{\partial X_{3}} & \frac{\partial N_{j}}{\partial X_{2}} \phi_{2} \\ 0 & 0 & N_{j} \frac{\partial \phi_{1}}{\partial X_{3}} & 0 & 0 & N_{j} \frac{\partial \phi_{2}}{\partial X_{3}}\end{array}\right]$

The stiffness matrix of the $k$-th lamina is shown in Eq. (11).

$K_{i j}^{(k)}=\int_{\Omega^{(k)}} \int_{Z_{b o t}^{(k)}}^{Z_{t o p}^{(k)}} B_{m i}^{(k)} C_{m n}^{(k)} B_{n j}^{(k)} \mathrm{d} z \mathrm{~d} \Omega^{(k)}$

\section{Boundary conditions and linear system}

One exceptional advantage of the finite element approach based on a layerwise displacement field is the flexibility to insert boundary conditions. Because the element is discretized in multiple independent layers, it is possible to apply the boundary conditions to a specific layer. To illustrate how to assemble the linear system and to apply boundary conditions with the layerwise approach, consider the case of Fig. 3, a fixed bar idealized as a single 1D element with two layers, with linear interpolation through 
Fig. 3 Illustrative case of a twolayer 1D element with linear layerwise interpolation through the thickness
Boundary conditions

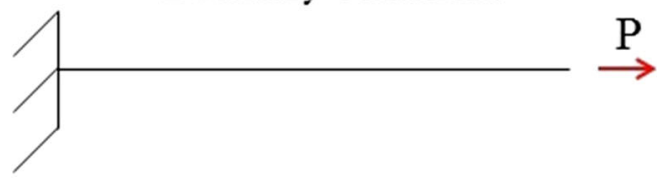

Idealized model

Node 1 $\left\{\begin{array}{l}u_{1}^{3}=U_{3} \\ u_{1}^{2}=U_{2} \\ u_{1}^{1}=U_{1}\end{array} \quad\right.$ Node 2 $\left\{\begin{array}{l}u_{2}^{3}=U_{6} \\ u_{2}^{2}=U_{5} \\ u_{2}^{1}=U_{4}\end{array}\right.$

Layers discretization

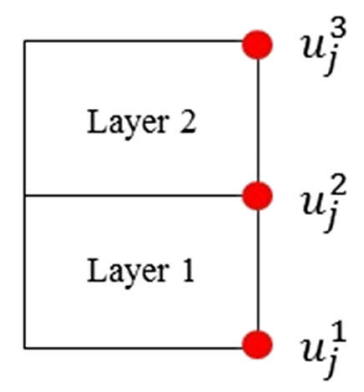

Fig. 4 Global linear system of the problem of Fig. 3

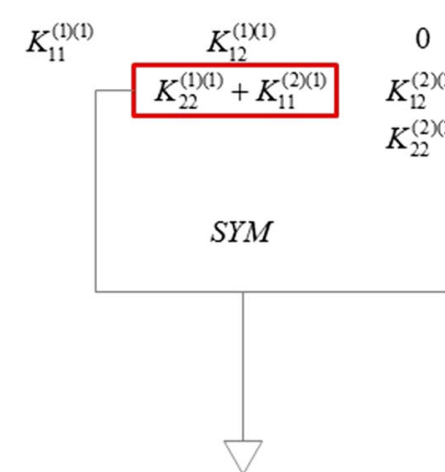

Compatibility imposed the thickness. Observe that the degrees of freedom in the local system are the variable $u_{j}^{k}$, where $j$ is the node and $k$ is the layer index, and the degree of freedom is expressed in the global system as $U$ (hence this case has six degrees of freedom).

To introduce the boundary conditions of the fixed end it is necessary to impose $U_{1}=U_{2}=U_{3}=0$; Restraining all the degrees of freedom of the fixed node is sufficient to restrict the rotation of it. If it is desired to insert a simple support, only the degrees of freedom related to the bottom plane should be forced to zero. The nodal force $P$ can be applied to the fourth to sixth positions of the force vector. $F_{4}=P$ means that the nodal force $P$ is being applied to the bottom plane of the laminate, $F_{5}=P$ implies that the nodal fore is at the interface between the layers and $F_{6}=P$ is at the upper plane of the laminate.

Equations (12) and (13) express the general vector of nodal parameters of a node cantilevered and with pinned support boundary conditions, respectively.

$$
\begin{aligned}
& u_{j}=\left[u_{1}^{(1)} u_{2}^{(1)} u_{3}^{(1)} u_{1}^{(2)} u_{2}^{(2)} u_{3}^{(2)} \ldots u_{1}^{(k)} u_{2}^{(k)} u_{3}^{(k)} u_{1}^{(k+1)} u_{2}^{(k+1)} u_{3}^{(k+1)}\right]_{j} \\
& =0
\end{aligned}
$$

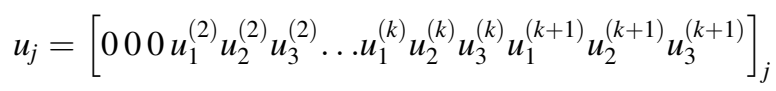

The linear system of the problem illustrated in Fig. 3 is expressed in Fig. 4. Observe that that compatibility of displacements at interfaces is imposed by assembling the contribution of the stiffness of the two adjacent laminas, as is emphasized in Fig. 4. The first superscript of the elemental stiffness matrix $K$ refers to the number of the lamina and the second is the number of the node. If there were more than one single element, then another index is necessary to represent the element number. 


\section{Homogenization}

Heterogeneous media are the composite mixtures of two or more constituents whose components are idealized to be bonded together. To proceed with a structural analysis of a reinforced deep beam with the layerwise theory it is necessary to homogenize the layers which contain both reinforcement and concrete. Note that the reinforcement is non-periodic; therefore it is not possible to use asymptotic homogenization methods. Hence the following non-periodic averaging methods are employed to calculate the elastic effective properties:

\subsection{The Voigt model}

Voigt et al. [14] adopted the iso-strain assumption to obtain the estimation of the longitudinal effective Young's modulus $E_{1}^{*}$ and effective Poissońs ratio $v_{12}^{*}$ as the weighted volume average of the stiffness matrices of constituent phases, i.e., as presented in Eq. (14),

$$
E_{1}^{*}=v_{m} E_{m}+v_{f} E_{f}
$$

$v_{12}^{*}=v_{m}+v_{f}$

where $E_{m}, E_{f}, v_{m}, v_{f}, v_{m}$ and $v_{f}$ are the Young's moduli, Poissońs ratios, and fraction volumes of the matrix and reinforcement, respectively. Note that: $v_{m}+v_{f}=1$

\subsection{The Reuss model}

Based on the iso-stress assumption Reuss [15] estimated the effective transverse Young's modulus $E_{2}^{*}$ and in-plane shear modulus $G_{12}^{*}$, written in Eq. (15) as the weighted volume average of the compliance matrices of constituent phases, i.e.,

$$
\begin{aligned}
E_{2}^{*} & =\frac{E_{m} E_{f}}{v_{f} E_{m}+v_{m} E_{f}} \\
G_{12}^{*} & =\frac{G_{m} G_{f}}{v_{f} G_{m}+v_{m} G_{f}}
\end{aligned}
$$

\subsection{Hill and Hashin models}

Hill [16] and Hashin [17] proposed a macroscopically homogeneous stress/strain field to represent the volume element to calculate the longitudinal effective properties, written as Eq. (16):

$$
\begin{aligned}
& E_{1}^{*}=v_{m} E_{m}+v_{f} E_{f}+\frac{4 v_{m} v_{f}\left(n_{f}-n_{m}\right)^{2} G_{m}}{\frac{v_{m} G_{m}}{K_{f}}+\frac{\sigma_{f}}{3}}+\frac{v_{f} G_{m}}{K_{m}+\frac{G_{m}}{3}}+1 \\
& \text { with } \quad K=E G / 3(3 G-E) \\
& v_{12}^{*}=v_{m} n_{m}+v_{f} n_{f}+\frac{v_{m} v_{f}\left(n_{f}-n_{m}\right)\left(\frac{G_{m}}{K_{m}+\frac{G_{m}}{3}}-\frac{G_{m}}{F_{f}+\frac{G_{f}}{3}}\right)}{\frac{v_{m} G_{m}}{K_{f}+\frac{G_{f}}{3}}+\frac{v_{f} G_{m}}{K_{m}+\frac{\sigma_{m}}{3}}+1}
\end{aligned}
$$

In the present work, the longitudinal effective properties are calculated by averaging the results obtained on Voigt and Hill-Hashing formulations [14-16], whereas the transverse properties are homogenized using the Reuss model [15]

\section{Numerical examples and discussion}

\subsection{First numerical example}

The first numerical example of this paper is a reinforced concrete deep beam with two supports, one pinned and the other simple. Concentrated loads of $90 \mathrm{kN}$ are applied to specific areas of $2809 \mathrm{~mm}^{2}$ at locations of the upper surface, resulting in a pressure of roughly $32 \mathrm{MPa}$. The geometric and elastic properties of the beams correspond to the 3BD50b beam in the investigation by Zhang and Tam [18]. A schematic drawing of the problem is shown in Fig. 5. In this model, the deep beam is considered to be in the linear phase.

In this layerwise model several layers are discretized through the thickness. The elastic properties of the layers containing both concrete and the reinforcements are homogenized using the averaging models described earlier. Three distinct analyses are performed using the layerwise formulation; the type of element used for all layerwise analyses is the 4-node homogenous element with linear layerwise interpolation through the thickness. All layerwise simulations are refined until convergence, resulting in 1449 elements with 1536 nodes. The number of virtual layers, however, differs in each analysis, resulting in more degrees of freedom (DoFs) in the analyses with more virtual layers. Table 1 displays the quantity of layers and DoFs in each analysis and Fig. 6 illustrates the discretization of the cross section to each case. The difference in the number of DoFs between layerwise and ABAQUS ${ }^{\mathrm{TM}}$ analyses is notable.

The elastic properties of the homogenized layers are written in MPa in Table 2, and the elastic properties in the ABAQUS $^{\mathrm{TM}}$ heterogeneous analysis are given in Table 3 . The material in the latter was modeled as isotropic for both concrete and for the steel bars. Furthermore, the elastic 

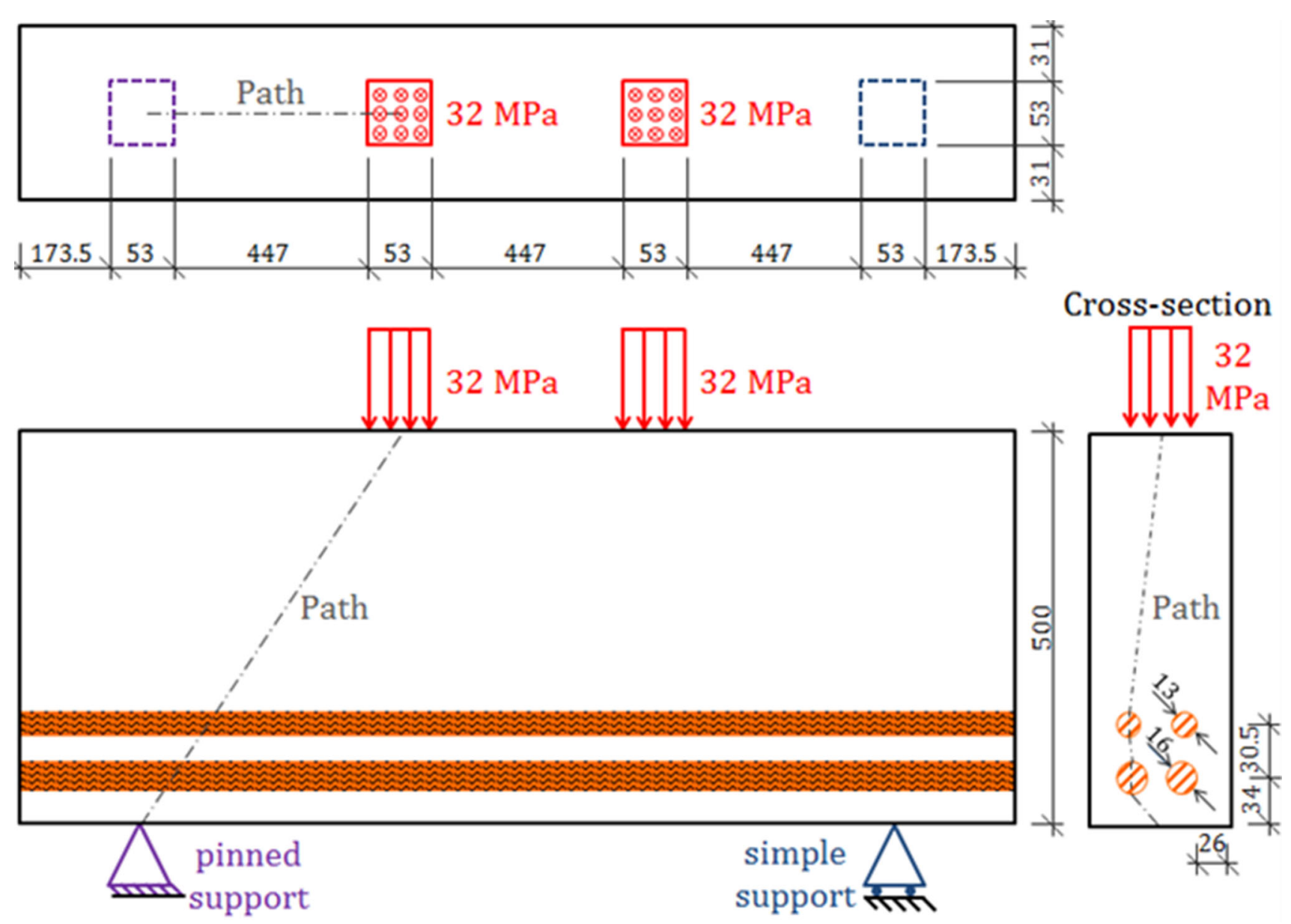

Fig. 5 Schematics of the first numerical example

Table 1 Discretization of each analysis

\begin{tabular}{|c|c|c|}
\hline & Number of layers & DoFs \\
\hline LW1 & 14 & 69,120 \\
\hline LW2 & 21 & 101,376 \\
\hline LW3 & 28 & 133,632 \\
\hline ABAQUS ${ }^{\mathrm{TM}}$ homogeneous & - & $1,003,860$ \\
\hline ABAQUS ${ }^{\mathrm{TM}}$ heterogeneous & - & $3,376,914$ \\
\hline
\end{tabular}

properties of the virtual layers with plain concrete on the layerwise analyses are the same as the pure concrete of Table 3.

The layerwise model proposed in this paper for deep beams is validated through ABAQUS ${ }^{\mathrm{TM}}$ simulations. Two models are carried out in $\mathrm{ABAQUS}^{\mathrm{TM}}$, as illustrated in Fig. 7a. In the first simulation (Fig. 7b) the layers containing both concrete and reinforcement are homogenized as discussed previously, and the type of element used is the 20-node brick element (element type C3D20). In the second ABAQUS ${ }^{\mathrm{TM}}$ analysis there is no homogenization, the
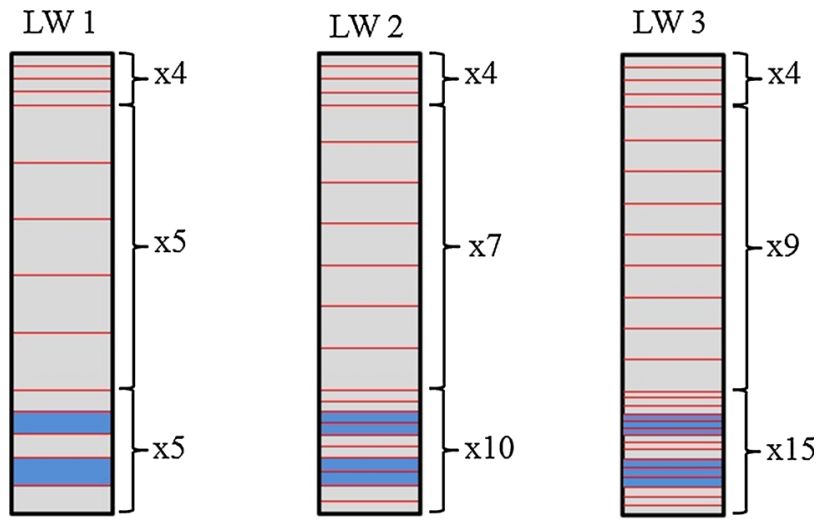

Fig. 6 Discretization of the cross section of each layerwise analysis

steel bars are manually designed with a cylindrical shape (Fig. 7c), and both concrete and steel bars are modeled as isotropic material perfectly bonded to each other. The element used in the latter is the hybrid 20-node brick element (element type C3D20H). It is assumed that the steel bars are completely enveloped by concrete and perfectly bonded to it. In addition, the stiffness of the steel is 
Table 2 Elastic properties in $\mathrm{MPa}$ of the virtual layers of the layerwise analyses

\begin{tabular}{llllllllll}
\hline & $E_{11}$ & $E_{22}$ & $E_{33}$ & $G_{12}$ & $G_{23}$ & $G_{13}$ & $v_{12}$ & $v_{23}$ & $v_{13}$ \\
\hline $\begin{array}{l}\text { Homogenized layers } \\
\varphi 16 \mathrm{~mm}\end{array}$ & $61,775.2$ & $30,399.3$ & $E_{22}$ & $12,243.5$ & $G_{12}$ & $G_{12}$ & 0.188 & $v_{12}$ & 0.241 \\
$\begin{array}{l}\text { Homogenized layers } \\
\varphi 13 \mathrm{~mm}\end{array}$ & $54,113.1$ & $29,098.1$ & $E_{22}$ & $11,702.9$ & $G_{12}$ & $G_{12}$ & 0.199 & $v_{12}$ & 0.243 \\
\hline
\end{tabular}

Table 3 Elastic properties in MPa of concrete and steel bars

\begin{tabular}{llcl}
\hline & $E$ & $G$ & $v$ \\
\hline Plain concrete & 24,602 & $9,840.9$ & 0.25 \\
Steel bars & 210,000 & 78,947 & 0.33 \\
\hline
\end{tabular}

considerably superior to the concrete's. Thus the response of the steel bars could be considered as incompressible, meaning a very small variation in displacement results in a large changes in pressure, so that a purely displacement- based solution is too sensitive to be useful numerically. The hybrid formulation of ABAQUSTM eliminates this singular behavior by using a mixture of displacement and stress variables with an increased variational principle to approximate the equilibrium equations and compatibility conditions.

The results are shown in Figs. 9, 10, 11 and 12; these graphics display all components of the stress and strain tensors that are different from zero within the strut. The path considered to compare stresses and strains in the homogeneous ABAQUS ${ }^{\mathrm{TM}}$ and layerwise models is along
Fig. 7 a Isometric view of the general geometry of the beam modeled in ABAQUS ${ }^{\mathrm{TM}}$, with boundary conditions; $\mathbf{b}$ front view of the homogeneous model; $\mathbf{c}$ front view of the heterogeneous model

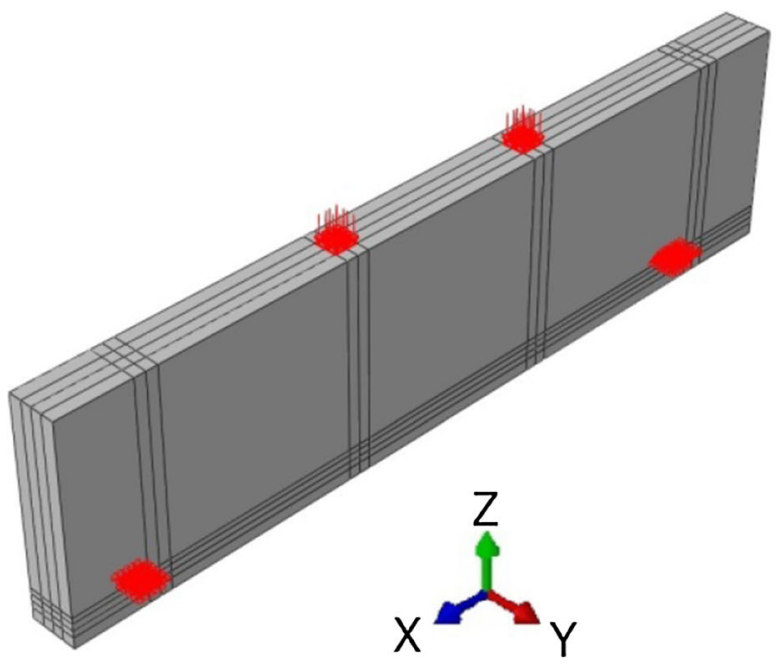

(a)

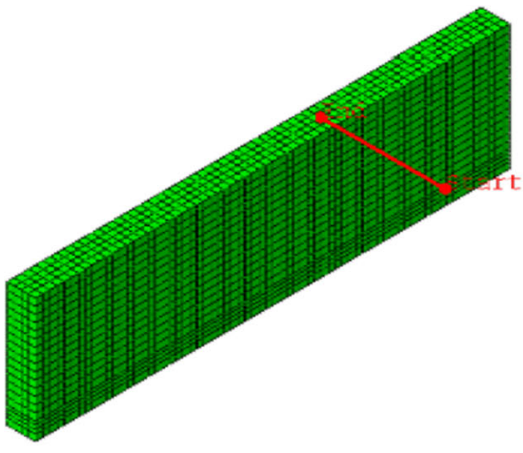

(a)

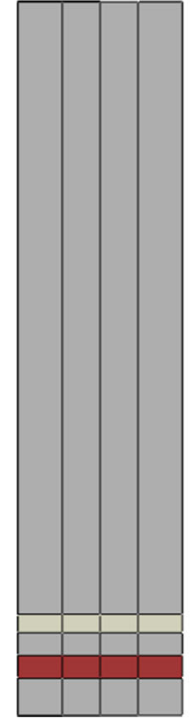

(b)

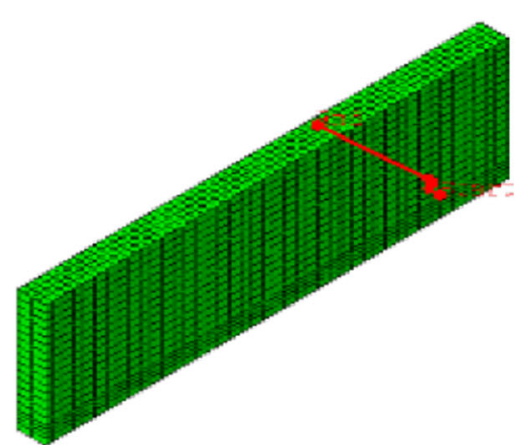

(b)

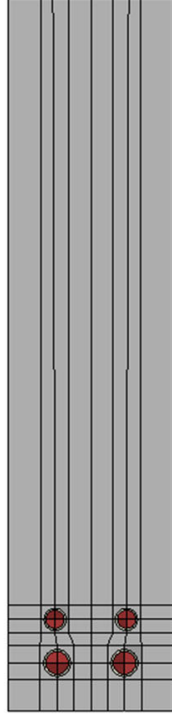

(c)

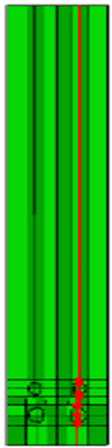

(c) 


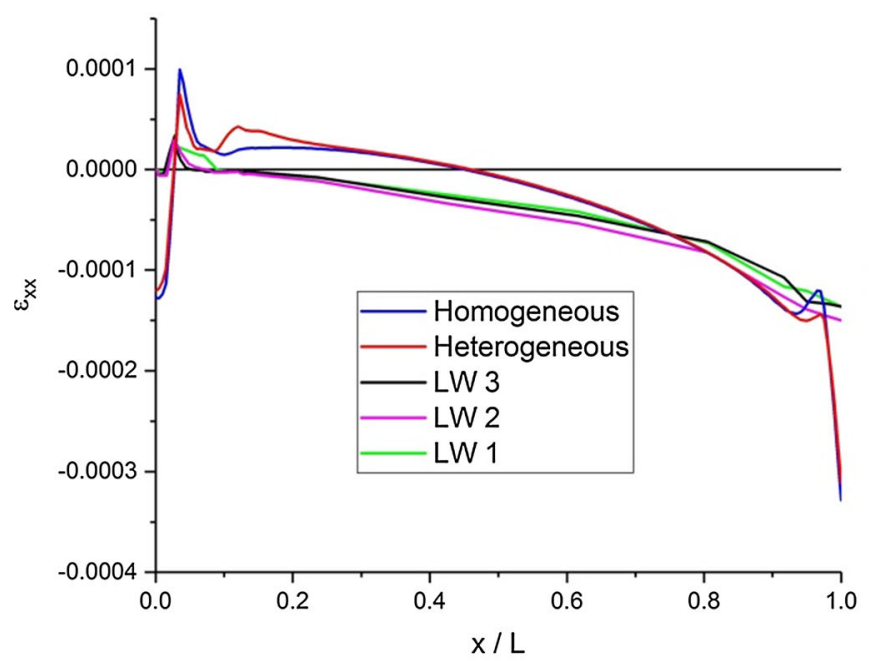

(a)

Fig. 9 a Normal strain in $\mathrm{xx}$ direction; $\mathbf{b}$ normal stress in $x x$ direction

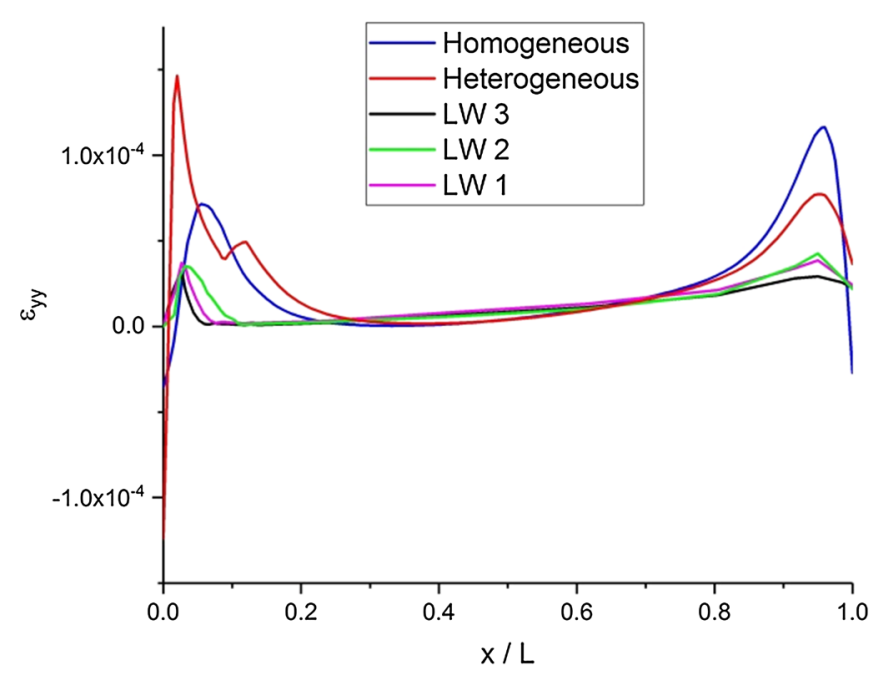

(a)

Fig. 10 a Normal strain in $y y$ direction; b normal stress in $y y$ direction

the strut, as illustrated in Fig. 8a. Regarding the heterogeneous ABAQUS ${ }^{\mathrm{TM}}$ model, the path crosses the center of the steel reinforcements, as shown in Fig. 8b. The curves named homogeneous are the results of the ABAQUS ${ }^{\mathrm{TM}}$ model with homogenization of layers, and those named heterogeneous are the results of the ABAQUS ${ }^{\mathrm{TM}}$ simulation with the reinforcement discretized. The layerwise curves correspond to the layerwise analyses, whose discretization through the thickness is already demonstrated in Fig. 6. As is shown in the results of Figs. 9, 10, 11 and 12, the more refined the cross section of the layerwise analyses, the more accurate are the results. However, the less refined model (LW1) also presents accurate results.

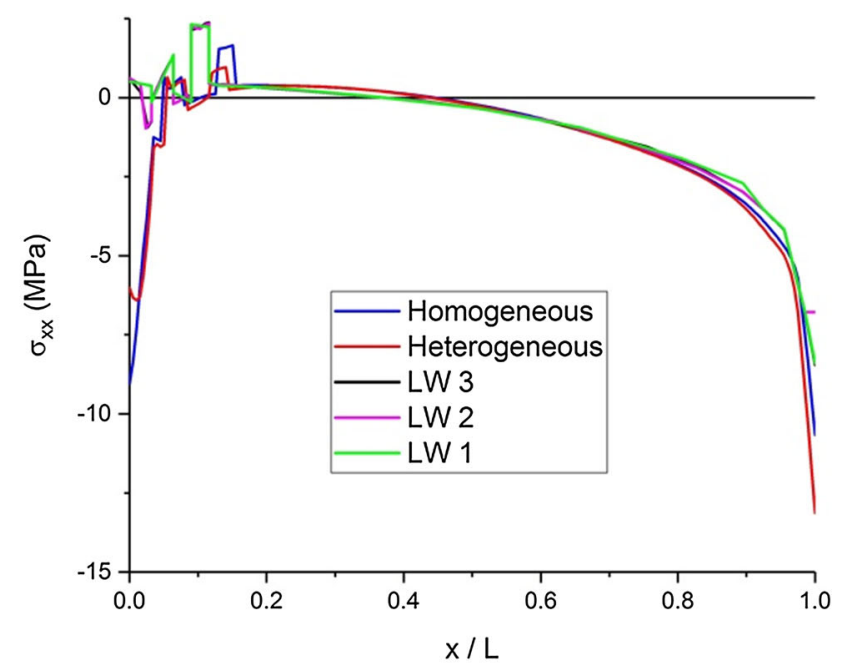

(b)

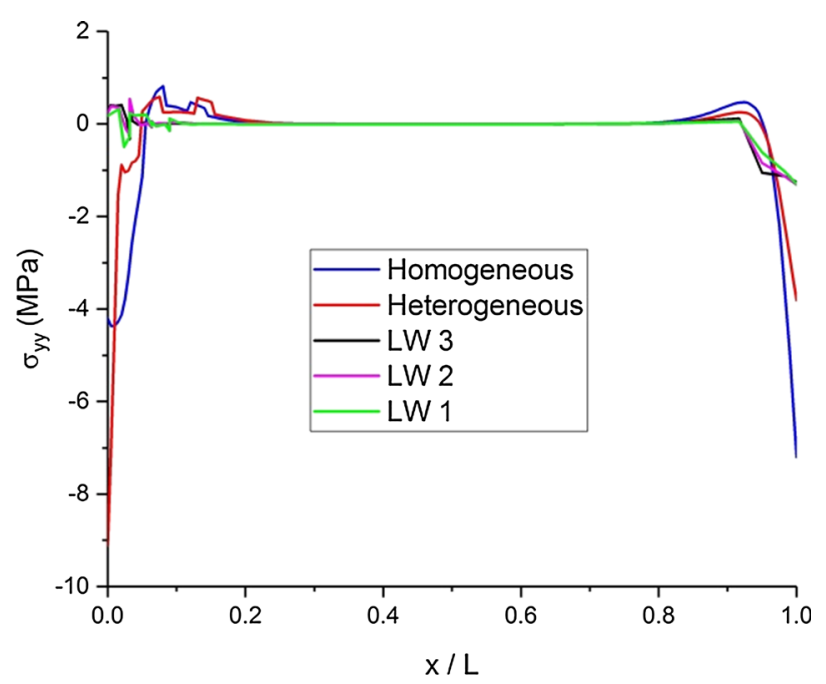

(b)

Figure 9 shows the behavior of stresses and strains in a longitudinal direction along the strut. As can be seen, there are perturbations at the bottom end of the strut. This phenomenon occurs due to the abrupt change in elastic properties between the reinforcement and the concrete, which is adequately presented in all curves. Furthermore, the layers with reinforcements are under tensile stress in the longitudinal direction, while the strut is compressed as expected.

Figure 10 is the out-of-plane component of the strain/ stress tensors. These two graphics show that the out-ofplane stress and strain are approximately zero all over the domain of the strut but not close to the boundary conditions domain, which is in accordance with Saint-Venant's 


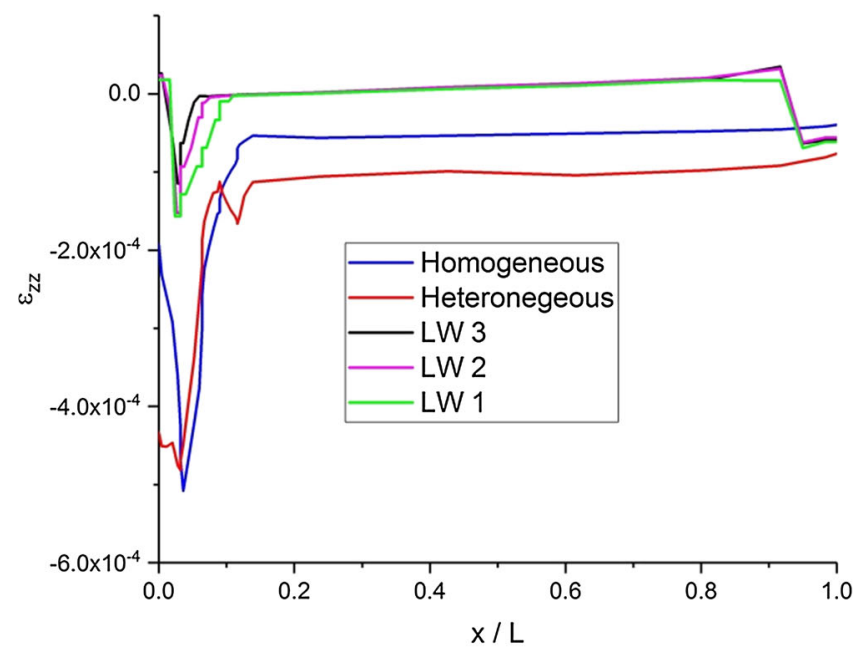

(a)

Fig. 11 a Normal strain in $z z$ direction; $\mathbf{b}$ normal stress in $z z$ direction

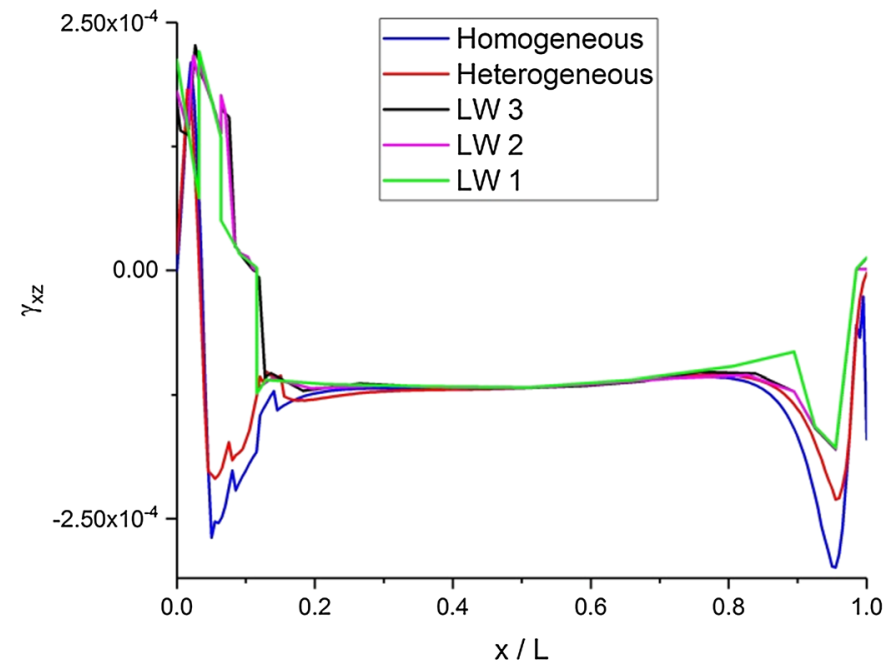

(a)

Fig. 12 a Shear strain in $x z$ direction; $\mathbf{b}$ shear stress in $x x$ direction

principle. All simulations point to a three-dimensional state of stress field near the boundary conditions.

Figure 11 is the strain and stress in the height direction. In the stress graphic all curves converge to the same value over the middle area of the strut, but not the strains. Furthermore, both layerwise and ABAQUS ${ }^{\mathrm{TM}}$ curves successfully capture the phenomenon of the discontinuity of the stress field at the interface between concrete and reinforcements. The discontinuity is more visible in layerwise curves, because since the approximation of the displacement field is linear, the discontinuity at the interfaces of stresses and strains is a vertical leap. On the other hand, the ABAQUS $^{\mathrm{TM}}$ approximation of the displacement field is parabolic; hence, the stress/strain discontinuity at interfaces in this direction is represented by a kink.

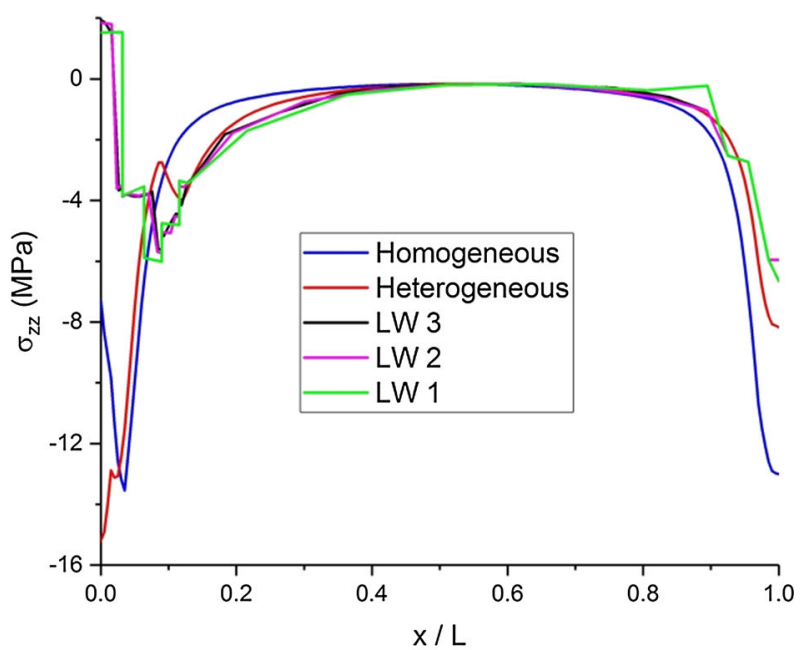

(b)

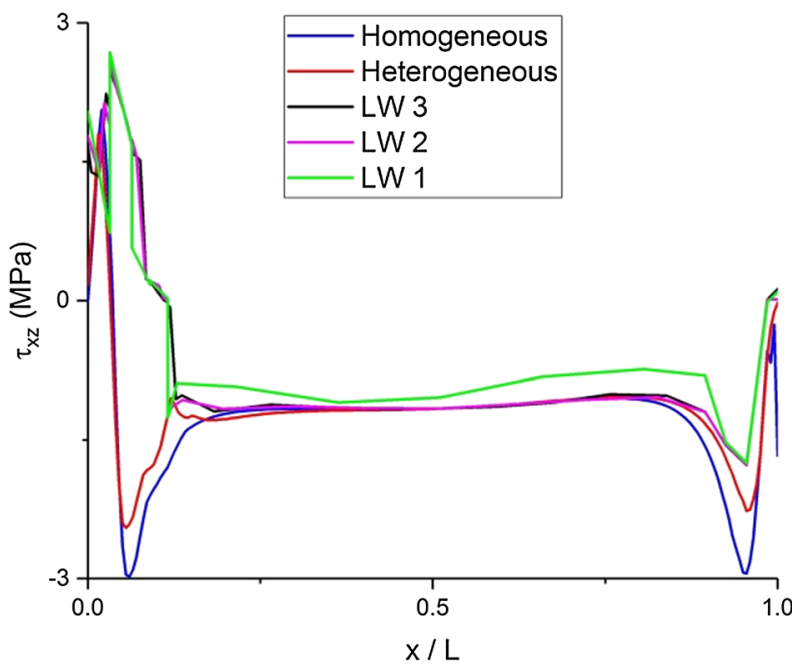

(b)

Figure 12 shows the behavior of in-plane shear stress and strain. Over the strut domain, all curves show that the shear strain converges to zero, but the shear stress is constant. At the boundaries the results are different but show the same trend. Moreover, all curves meet the natural boundary condition of free shear strain/stress at both ends.

\subsection{Second numerical example}

The second numerical model is a homogenous deep beam with both ends fixed and a constant pressure on top. Figure 13 illustrates the boundary conditions, and geometrical and elastic properties of this numerical example. The third dimension of the beam (perpendicular to the plane) was considered to be unitary. The mesh has 100 elements and 


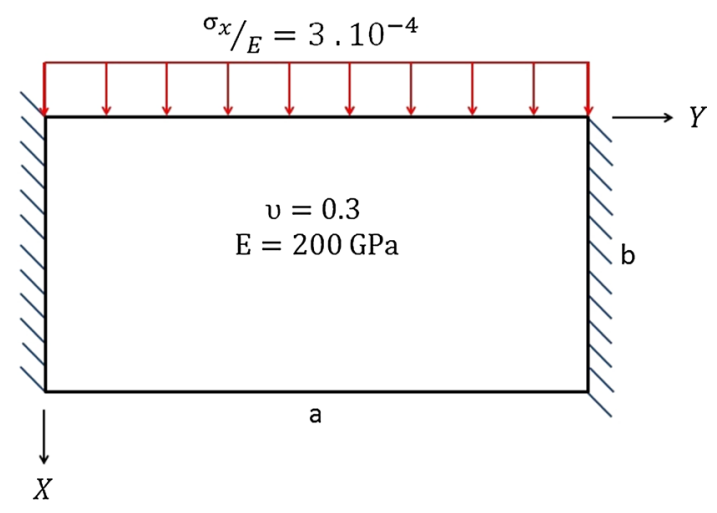

Fig. 13 Homogeneous deep beam with both ends cantilevered, solved by Ahmed and Idris [6]

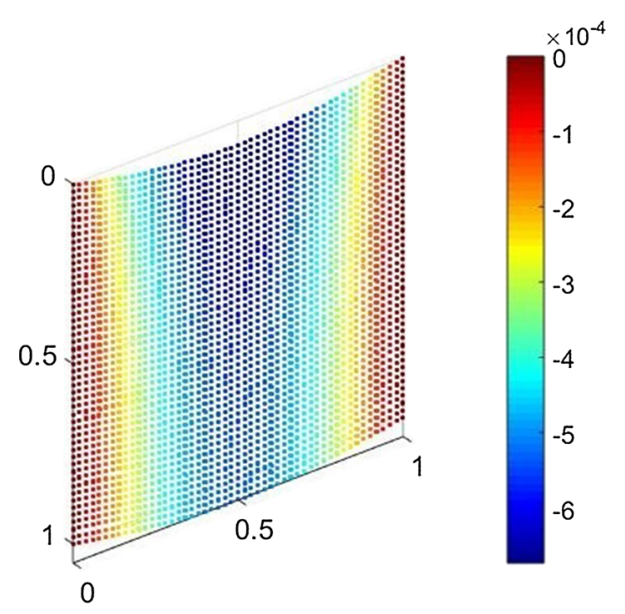

Fig. 14 Gradient of the displacement in $\mathrm{x}$ direction

50 layers are discretized across the $x$ direction, resulting in 23,409 DoFs. Figure 14 shows the gradient of displacements in the pressure direction ( $X$ direction) normalized by the $b$ dimension. The results are compared to Ahmed and Idris [6], who solved the same problem through a 2D numerical approach based on the finite-difference method.
Figures 15, 16, 17 and 18 show the distribution of displacement and stresses in different sections and geometry. In order to make the results non-dimensional, the displacements are normalized by using the dimension $b$, and the stresses are normalized by Young's modulus.

Figure 15 is the distribution of displacement $u$ ( $X$ direction) at different sections. Observe the parabolic nature and that the displacement $u$ becomes higher near the top edge and is at its minimum near the bottom edge. The distribution of displacement $v$ ( $Y$ direction) of Fig. 16 shows the sinusoidal variation of the top plane, which is also in accordance with the physical model of the problem. It is observed that the sinusoidal behavior is more pronounced for bigger $a / b$ relations. Furthermore, at both ends the displacement converges to zero due to the applied boundary conditions.

Figure 17 displays the distribution of normal stress in longitudinal direction at the restrained boundaries for different $a / b$ relations. Observe that the normal stress $\sigma_{y}$ changes from positive to negative at $x / b=0.5$, which means a change from compressive stress to tensile. One can observe the non-symmetry of the finite element solution, which does not agree with Ahmed and Idris's [6] results. This divergence can be explained by the fact that the procedure carried out here considers a complete state of stress, while the finite-difference solution of [6] solution admits an in-plane state of stress.

The distribution of shear stress displayed in Fig. 17 shows the nonlinear behavior at the cross section near the mid-section. For lower $a / b$ relations the curves become closer to uniform distribution, and the shear stress vanishes at the midpoint.

Lastly, Figs. 19 and 20 show the behavior of out-ofplane stress. It is possible to conclude by analyzing Fig. 19 that the out-of-plane stress tends to zero over all domains of the deep beam, but not at the boundaries. At both ends the out-of-plane stress has the same magnitude as the other
Fig. 15 Displacement $u$ for different sections for $a / b=2$; a layerwise theory; b Ahmed and Idris [6]

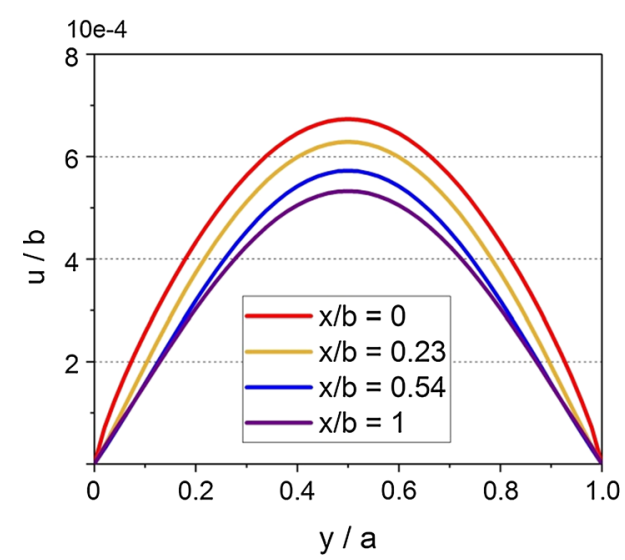

(a)

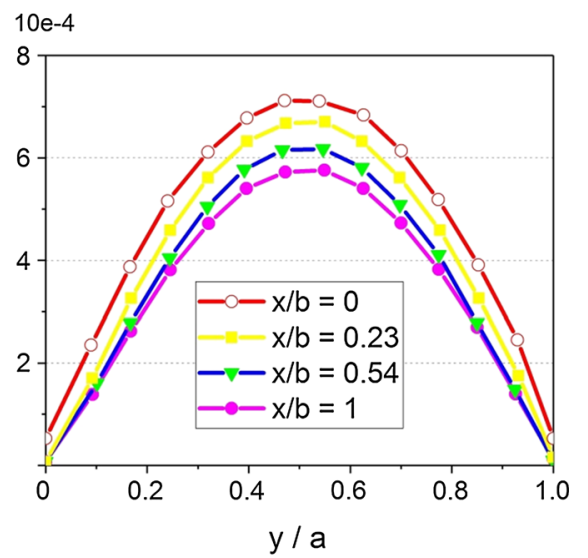

(b) 
Fig. 16 Displacement $v$ at section $x / b=0$ for different $a / b$; a layerwise theory; b Ahmed and Idris [6]
Fig. 17 Stress $\sigma_{y}$ at section $y /$ $a=0$ for different $a / b$; a layerwise theory; b Ahmed and Idris [6]

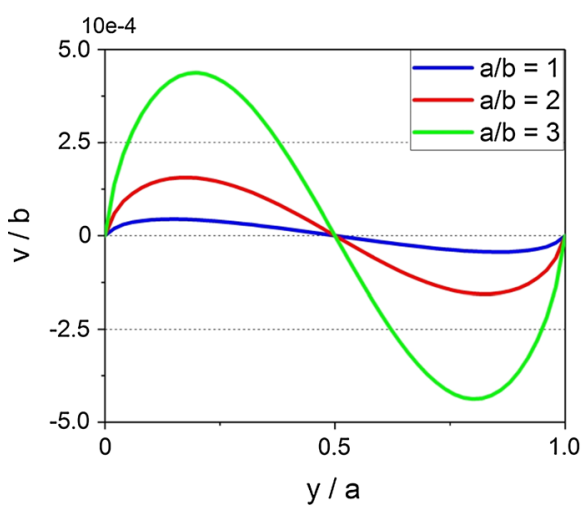

(a)

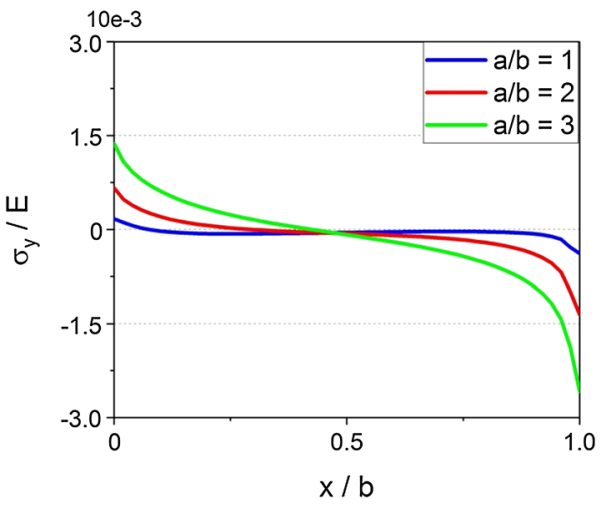

(a)

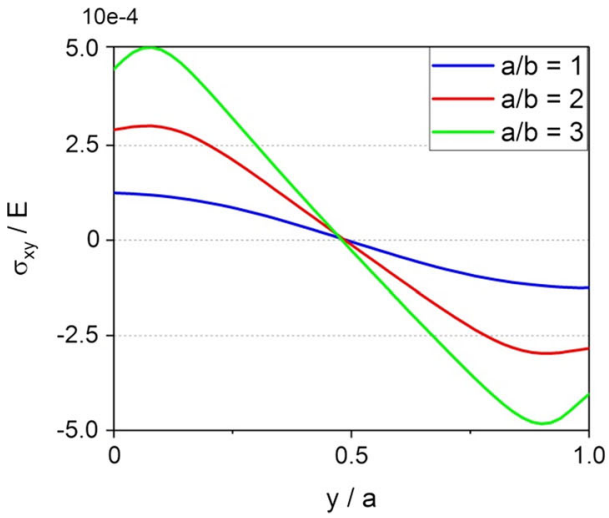

(a)

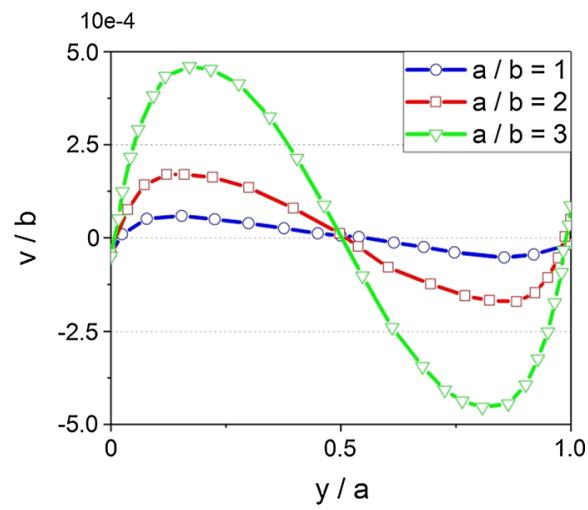

(b)

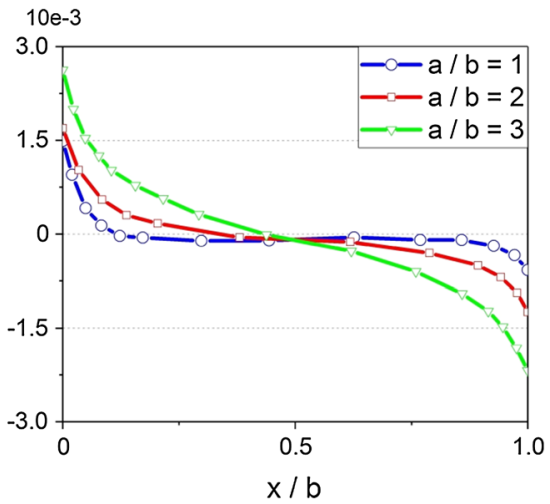

(b)

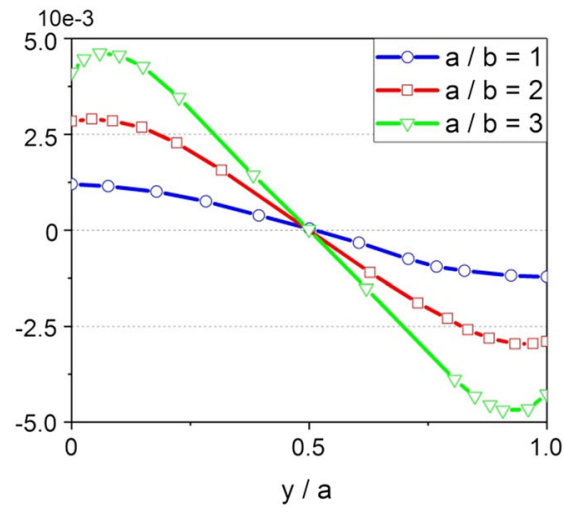

(b) components of the stress tensor. Figure 20 shows the behavior of the out-of-plane stress throughout the cantilevered section for different geometries, and it is visible that the stress tends to change from tensile to compressive at the middle plane. An interesting fact observed in Fig. 20 is that a quadrilateral geometry soothes the out-of-plane normal stress, resulting in nearly zero out-of-plane normal stress all over the beam domain.

\section{Conclusion}

In this paper a finite element procedure based on the layerwise displacement was suggested to perform structural analyses of deep beams. Two numerical examples were proposed: one reinforced concrete deep beam, validated by similar analysis in $\mathrm{ABAQUS}^{\mathrm{TM}}$ software, and one isotropic deep beam with both ends fixed, validated by former finitedifference analysis in [6]. 


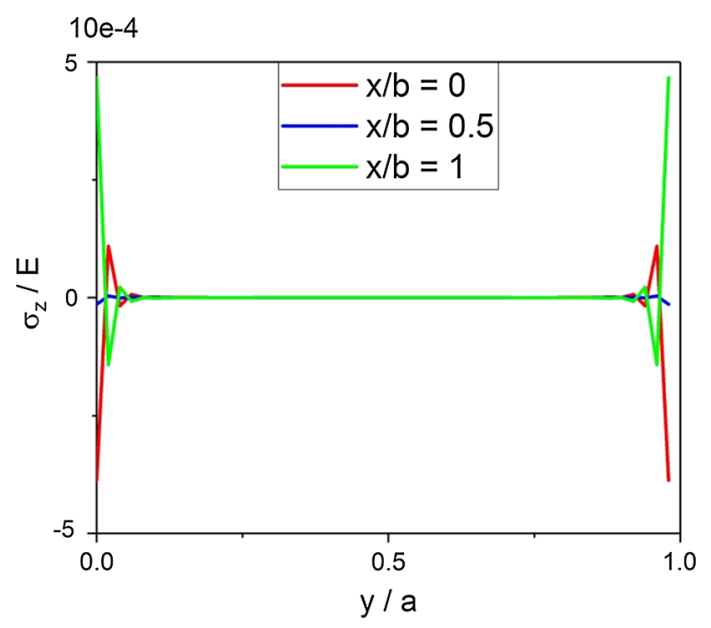

Fig. 19 Out-of-plane stress for $a / b=2$ for different sections

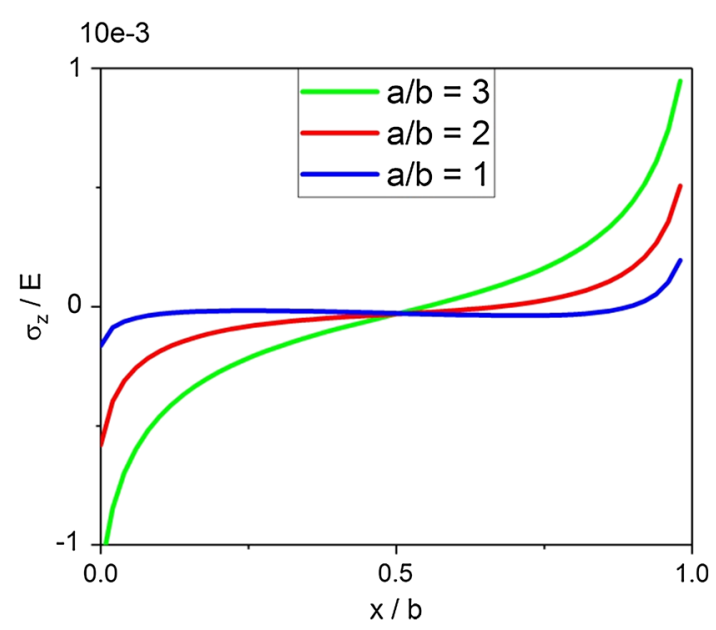

Fig. 20 Out-of-plane stress for section $y / a=0$ for different $a / b$

In the reinforced concrete numerical example, both models carried out in $\mathrm{ABAQUS}^{\mathrm{TM}}$ presented similar results. The heterogeneous and homogenized curves of Figs. 9, 10, 11 and 12 were roughly the same. In fact, one should even considerer opting for the homogenized analysis instead of the heterogeneous one due to the lower computational effort required. The layerwise analyses were precise; all three layerwise analyses of Figs. 9, 10, 11 and 12 generated similar curves; hence there was no need to discretize too many layers. Furthermore, the layerwise solutions were in agreement with the ABAQUS ${ }^{\mathrm{TM}}$ results. However, close to the boundary conditions the layerwise and $\mathrm{ABAQUS}^{\mathrm{TM}}$ formulations slightly diverged. It is important to remark that the analysis performed in this first numerical example considered the reinforced concrete in its linear phase. In order to extend this analysis to linearplastic, one could apply continuum damage mechanics basics to specific layers, or employ another constitutive relation.
Regarding the second numerical example, Figs. 15, 16, 17 and 18 show that the solution of the layerwise approach and the solution of [6] match each other. Although, Figs. 19 and 20 reveal that the current analysis should idealize a tridimensional state of stress over the cantilevered edges because the out-of-plane stress component has a pronounced magnitude.

The advantages of the layerwise approach for performing structural analyses of heterogeneous deep beams are manifold. First, the layerwise formulation is capable of carrying out full 3D analysis with less computational effort than equivalent analyses based on solid elements. In addition, the layerwise formulation is more adequate to approach heterogeneous structures than SDT because it represents more correctly the stress/strain field at the interfaces of different materials. Finally, the layerwise theory is free of shear and membrane locking, and is able to simulate complex problems that are not possible in STM models.

Acknowledgements The authors acknowledge the financial support of Coordenação de Aperfeiçoamento de Pessoal de Nível Superior (CAPES)-PROEX, and two scholarships (Process Numbers: DS00011/07-0 and PDSE99999.007060/2015-07), as well as the financial support of FUSP (Process Number: 3016/TT-4) and Fundação de Amparo à Pesquisa do Estado de São Paulo (FAPESP) to project 2015/13844-8.

\section{References}

1. ACI Committee 318 (2008) Building code requirements for structural concrete and commentary. ACI 318-08/ACI 318R-08. American Concrete Institute, Farmington Hills, p 465

2. Schlaich J, Schafer K, Jennewein M (1987) Toward a consistent design of structural concrete. PCI J 32:74-150. https://doi.org/10. 15554/pcij.05011987.74.150

3. Russo G, Venir R, Pauletta M, Somma G (2005) Reinforced concrete corbels-shear strength model and design formula. ACI Struct J 103:3-10. https://doi.org/10.14359/15080

4. Mihaylov BI, Bentz EC, Collins MP (2013) Two-parameter kinematic theory for shear behavior of deep beams. ACI Struct J 110:447-455. https://doi.org/10.14359/51687180

5. Liu J, Mihaylov BI (2016) A comparative study of models for shear strength of reinforced concrete deep beams. Eng Struct 112:81-89. https://doi.org/10.1016/j.engstruct.2016.01.012

6. Ahmed SR, Idris ABM, Uddin MW (1996) Numerical solution of both ends fixed deep beams. Comput Struct 61:21-29. https://doi. org/10.1016/0045-7949(96)00029-6

7. Ahmed SR, Khan MR, Islam KMS, Uddin MW (1998) Investigation of stresses at the fixed end of deep cantilever beams. Comput Struct 69:329-338. https://doi.org/10.1016/s00457949(98)00127-8

8. Reddy JN (1987) A generalization of two-dimensional theories of laminated composite plates. Commun Appl Numer Methods 3:173-180. https://doi.org/10.1002/cnm.1630030303

9. Liu D, Li X (1996) An overall view of laminate theories based on displacement. J Mater Sci 30:1539-1561. https://doi.org/10.1177/ 002199839603001402 
10. Reddy JN (1989) On refined computational models of composite laminates. Int J Numer Methods Eng 27:361-382. https://doi.org/ $10.1002 / \mathrm{nme} .1620270210$

11. Reddy JN (2004) Mechanics of laminated composite plate and shells: theory and analysis, 2nd edn. CRC Press LLC, Florida, pp 725-820

12. Demasi L (2009) Mixed plate theories based on the generalized unified formulation. Part I: governing equations. Compos Struct 87:1-11. https://doi.org/10.1016/j.compstruct.2008.07.013

13. Nguyen DT (2012) Benchmark d'un modèle layer wise de multicouches et implémentation du modèle dans ABAQUS ${ }^{\mathrm{TM}}$. Université Paris-Est

14. Voigt W (1889) Ueber die Beziehung zwischen den beiden Elasticitätsconstanten isotroper Körper. Ann Phys 274:573-587. https://doi.org/10.1002/andp.18892741206
15. Reuss A (1929) Berechnung der Fließgrenze von Mischkristallen auf Grund der Plastizitätsbedingung für Einkristalle. ZAMM Zeitschrift Für Angew Math Und Mech 9:49-58. https://doi.org/ 10.1002/zamm.19290090104

16. Hill R (1964) Theory of mechanical properties of fibrestrengthened materials: I. Elastic behaviour. J Mech Phys Solids 12:199-212. https://doi.org/10.1016/0022-5096(64)90019-5

17. Hashin Z (1966) Viscoelastic fiber reinforced materials. AIAA J 4:1411-1417

18. Zhang N, Tan KH (2007) Size effect in RC deep beams: experimental investigation and STM verification. Eng Struct 29:3241-3254. https://doi.org/10.1016/j.engstruct.2007.10.005 